\title{
RELAÇÃO ENTRE AS ANOMALIAS DE TSM E QUALIDADE POTENCIAL DA UVA NA REGIÃO NORDESTE DO RIO GRANDE DO SUL ${ }^{1}$
}

\author{
JULIANO LISBÔA GRUPPELLI², JULIO RENATO MARQUES ${ }^{3}$,GILBERTOBARBOSADINIZ ${ }^{4}$
}

RESUMO-Uma das culturas de grande importância para a economia do Rio Grande do Sul é a videira. Embora as produções de vinho, suco de uva e demais derivados da uva e do vinho também ocorreram em outras regiões, a maior concentração encontra-se na região nordeste do Rio Grande do Sul. Um dos principais fatores que influenciam na qualidade potencial da uva é a precipitação. Para a videira, influem não somente a quantidade total de chuvas, mas também sua distribuição ao longo do ciclo vegetativo. Por esses motivos, é de fundamental importância o estudo da precipitação na região nordeste do Estado. Dentre as variáveis pesquisadas que relacionam a variabilidade da precipitação, destaca-se a Temperatura da Superfície do Mar (TSM). Os objetivos principais deste trabalho são: identificar os padrões de anomalias da TSM dos oceanos Atlântico e Pacífico e suas relações na precipitação, na região nordeste do Rio Grande do Sul, nos meses de outubro a março. Dessa forma, procurou-se investigar a possibilidade de uma previsibilidade da precipitação com alguns meses de antecedência e, conseqüentemente, uma possível previsão da qualidade potencial da uva para aquela região. Os resultados mostraram que o primeiro padrão principal de sete áreas oceânicas apresentou correlação altamente significativa com a precipitação acumulada de janeiro e fevereiro nesta região e, conseqüentemente, uma correlação com a qualidade potencial da uva. Também ficou evidente a alta previsibilidade do padrão predominante nos meses de janeiro e fevereiro, a partir de anomalias da TSM média de outubro-novembro.

Termos para indexação: Variabilidade da precipitação, qualidade potencial da uva, anomalia de TSM.

\section{RELATIONSHIP BETWEEN OCEANIC INDICATORS AND POTENTIAL QUALITY OF GRAPE IN THE NORTHEAST REGION OF RIO GRANDE DO SUL}

\begin{abstract}
One of the cultures of great importance for the economy of Rio Grande do Sul is the grapevine. Although the productions of wine, grape juice and other products derived from the grape and the wine also occur in other regions, the biggest concentration is found in the northeast region of Rio Grande do Sul. Among the main factors that influence the potentiality of the grape is the precipitation. For the grapevine not only influences the total amount of rains, but also its distribution throughout the vegetative cycle. For these reasons it is of basic importance the study of the precipitation in the northeast region of the State. Among the variables researched which relate the variability of precipitation there is the sea surface temperature (SST). The main purposes of this work are: to identify the standards of anomalies of the SST of the Atlantic and Pacific oceans and their relations in the precipitation in the northeast region of Rio Grande do Sul in the months from October to March. Therefore, it was focused the investigation of a possible previsibility of the precipitation with some months of antecedence and consequently a possible forecast of the potentiality of the grape for that region. The results have shown that the first main standard of seven oceanic areas presented a highly significant correlation with the accumulated precipitation of January and February in this region, and consequently, a correlation with the qualitative potentiality of the grape. Also, the high previsibility of the predominant standard in the months of January and February from anomalies of the SST average of October-November was evident.
\end{abstract}

Index terms: Variability of precipitation, potential quality of grape, anomalies of SST.

\section{INTRODUÇÃO}

A economia da região Sul é baseada principalmente na agricultura, com aproximadamente $57 \%$ da produção nacional de grãos. Baseia-se também na pecuária, na geração de energia e nas indústrias, sendo essas atividades bastante suscetíveis às variabilidades climáticas e dependentes da quantidade e distribuição da precipitação (Berlato, 1992).

É comum no Estado ocorrerem períodos com elevada precipitação, bem como períodos de escassez. Esses períodos de grandes oscilações ocasionam riscos à agricultura, como, por exemplo, na qualidade potencial da uva da serra do nordeste gaúcho.

Uma das culturas de grande importância para a economia do Rio Grande do Sul é a videira. Embora as produções de vinho, suco de uva e demais derivados da uva e do vinho também ocorram em outras regiões, a maior concentração encontra-se no Rio Grande do Sul, onde são elaborados, anualmente, em média, 330 milhões de litros de vinhos e mostos, representando $95 \%$ da produção nacional. O clima possui forte influência sobre a videira, sendo importante na definição das potencialidades das regiões. Ele interage com os demais componentes do meio natural, em particular com o solo, assim como com a cultivar e com as técnicas

${ }^{1}$ (Trabalho 082-07). Recebido em: 28-03-2007. Aceito para publicação em: 07-01-2008.

${ }^{2}$ Meteorologista Mestre Climatologia, Lab. Climatologia/Faculdade de Meteorologia/UFPel. - UNIVERSIDADE FEDERAL DE PELOTAS

${ }^{3}$ Prof. Dr. Climatologia, Lab. Climatologia/Faculdade de Meteorologia/UFPel. - UNIVERSIDADE FEDERAL DE PELOTAS

${ }^{4}$ Prof. Dr. Climatologia, Lab. Climatologia/Faculdade de Meteorologia/UFPel. - UNIVERSIDADE FEDERAL DE PELOTAS 
agronômicas de cultivo. A precipitação pluviométrica é um dos elementos meteorológicos mais importantes na viticultura, sendo esta uma cultura bastante resistente à seca. Para a videira, influem não somente a quantidade total de chuvas, mas também sua distribuição ao longo do ciclo vegetativo. Por esses motivos, é de fundamental importância o estudo da precipitação na região nordeste do Estado.

Vários estudos fornecem evidências de que os oceanos Atlântico e Pacífico desempenham um papel significativo nas flutuações climáticas que ocorrem na região Sul do Brasil. A interação entre os oceanos e a atmosfera tem estimulado pesquisas no sentido de buscar as possíveis relações com os impactos das variações climáticas no meio ambiente. Entender qual o papel relativo dos oceanos no clima global, em diferentes escalas de tempo, ainda encontra-se em fase exploratória.

As relações mais claras das interações entre oceano e atmosfera aparecem nos grandes eventos do fenômeno El Niño Oscilação Sul (ENOS). O El Niño é um fenômeno atmosféricooceânico caracterizado por um aquecimento anômalo das águas superficiais no oceano Pacífico Tropical e que pode afetar o clima regional e global, mudando os padrões de vento em nível mundial, afetando, assim, os regimes de chuva em regiões tropicais e de latitudes médias.

Diaz et al. (1998) mostraram a existência de relações significativas entre as anomalias de precipitação sobre o Uruguai e o Rio Grande do Sul, e as anomalias de TSM dos oceanos Pacífico e Atlântico. Diaz e Studzinski (1994) estudaram a influência das TSM, do Atlântico Sul e do Pacífico Equatorial nas precipitações pluviais da região Sul do Brasil, encontrando uma boa coerência espacial entre as anomalias de TSM, principalmente para o período de outubro a dezembro, com maior homogeneidade na região mais ao sul. Marques (2005) mostrou que, durante o período da primavera, a precipitação no Rio Grande do Sul apresenta maior relação com a TSM do oceano Pacífico, Equatorial, mas, durante o verão, é a TSMs do Atlântico Subtropical que apresenta maior relação com a precipitação no Estado.

Dessa forma, o trabalho aqui desenvolvido tem como objetivo principal identificar os padrões de anomalias da TSM dos oceanos Atlântico e Pacífico, suas relações na precipitação na região nordeste do Rio Grande do Sul, nos meses de outubro a março, no período de 1982 a 2005, e analisar a possibilidade da previsibilidade da precipitação com alguns meses de antecedência e, conseqüentemente, uma possível previsão da qualidade potencial da uva na região nordeste do Rio Grande do Sul.

\section{MATERIAL E MÉTODOS}

Foram utilizados dados de precipitação mensal de outubro a março de 25 estações meteorológicas distribuídas no Estado, período de 1982 a 2005, pertencentes ao $8^{\circ} \mathrm{DISME} /$ INMET (Distrito de Meteorologia do Instituto Nacional de Meteorologia) e FEPAGRO-RS (Fundação Estadual de Pesquisa Agropecuária do Estado do Rio Grande do Sul).

O conjunto de dados de TSM utilizados foi obtido junto ao NCEP (National Center for Environmental Prediction) e NCAR (National Center for Atmospheric Research Reanalysis), numa grade regular de $1^{0} \times 1^{0}$ no formato NetCDF (Network Common Data Form), período de 1982 a 2005, abrangendo todo o oceano Pacífico Sul e Atlântico Sul (latitudes entre $10^{\circ} \mathrm{N} \mathrm{a} 60^{\circ} \mathrm{S}$ e longitudes de $120^{\circ} \mathrm{E}$ a $\left.30^{\circ} \mathrm{E}\right)$.

Inicialmente, foram calculados os escores dos componentes principais da precipitação, os quais foram obtidos pela técnica de decomposição da matriz correlação em autovalores e autovetores. Os escores são resultados do produto matricial dos autovetores e matriz padronizada dos dados de precipitação. As regiões homogêneas de precipitação no Rio Grande do Sul foram definidas pelo método de classificação K-means. O critério utilizado neste método foi a distância Euclidiana derivada dos quatro primeiros escores dos componentes.

O Método de Agrupamento consiste em escolher inicialmente o número de classes (5 classes), as quais são formadas pelo centro de massa da variável a ser agrupada, no caso, os escores dos componentes.

Após a definição das regiões homogêneas foi escolhida a região definida como nordeste. Para essa região, foram calculadas as médias mensais da precipitação regional dos anos de alto e baixo potenciais qualitativos da uva, considerando os meses de outubro a março, no período de 1982 a 2005.

As safras de alto e baixo potenciais da uva na região nordeste do Rio Grande do Sul foram escolhidas de forma qualitativa, segundo as categorias de classificação definidas por Mandelli et al. (2006). Westphalen (1977), analisando as condições climáticas do período de maturação das uvas, propôs o quociente heliopluviométrico de maturação (QM), que é a relação entre a insolação e a precipitação pluviométrica decorrentes do período que se deseja estudar. Mandelli et al. (2006) utilizaram dados de insolação e precipitação pluviométrica para classificar as condições climáticas do período de maturação da uva das safras de 1977 a 2003, da região de Bento Gonçalves. Os autores calcularam o QM no período efetivo (40 dias) de influência sobre a maturação da uva, utilizando as seguintes categorias de classificação das safras: alto potencial $(\mathrm{QM}>2)$; médio potencial (QM entre 1,0 e 2,0); baixo potencial $(\mathrm{QM}<1,0)$.

Foram identificados os meses de maior diferença entre as climatologias da precipitação regional para as safras de alta e baixa qualidade potencial. A precipitação regional do período identificado como o de maior importância foi relacionada com as anomalias de TSM. As identificações das áreas oceânicas de maior importância foram obtidas através do coeficiente de correlação. Inicialmente, foram feitas correlações com a TSM média do Niño 3.4; posteriormente, esse procedimento foi feito para as demais áreas dos oceanos Atlântico e Pacífico, buscando melhor explicar as variações temporais da precipitação neste período.

Por fim, foram verificadas as relações conjuntas de todas as áreas oceânicas que apresentaram coeficiente de correlação significativo (1\%), sendo representada pelo escore do primeiro componente principal destas áreas. 


\section{RESULTADOS E DISCUSSÃO}

A Figura 1a mostra o resultado do método de classificação K-means derivado dos quatro primeiros componentes da precipitação, definidas as 5 regiões homogêneas para o Rio Grande do Sul. Foi escolhida apenas uma região homogênea, a qual foi definida como região nordeste e está representada na Figura $1 b$.

Um dos fatores de escolha dessa região foi o fato de a mesma coincidir com a região de maior produção de uvas no Brasil, sendo o estudo desta cultura muito importante para a economia do Estado. Essa região está centrada, aproximadamente, na latitude $29^{\circ} \mathrm{S}$, longitude $51^{\circ} \mathrm{W}$ e altitude $600-800 \mathrm{~m}$. Seus indicadores climáticos médios são: precipitação na ordem de $1.700 \mathrm{~mm}$ distribuídos ao longo do ano, temperatura média anual em torno de $17,2^{\circ} \mathrm{C}$ e umidade relativa do ar média anual de $76 \%$.

A região nordeste é representada por 7 estações meteorológicas (Bento Gonçalves, Bom Jesus, Caxias do Sul, Farroupilha, Lagoa Vermelha, Torres e Veranópolis), sendo que a precipitação neste trabalho foi tratada de forma regional.

A Figura 2 mostra a climatologia mensal de precipitação da região nordeste do Rio Grande do Sul, onde se percebe claramente que as maiores diferenças de precipitação entre os anos de alto e baixo potenciais da uva apresentam maiores evidências nos meses de janeiro e fevereiro. Para os anos de alto potencial da uva, o mês de janeiro apresentou uma climatologia mensal da precipitação em torno de $96,9 \mathrm{~mm}$, e a média mensal regional de precipitação, em torno de $154,6 \mathrm{~mm}$, com uma diferença de $57,7 \mathrm{~mm}$ abaixo da média mensal regional. Para o mês de fevereiro, a climatologia mensal da precipitação foi em torno de $88,2 \mathrm{~mm}$, e a média mensal regional de precipitação, em torno de $145,1 \mathrm{~mm}$, com uma diferença de 56,9 $\mathrm{mm}$ abaixo da média. Em anos de baixo potencial da uva, o mês de janeiro mostrou uma climatologia mensal da precipitação, em torno de $211,1 \mathrm{~mm}$, e a média mensal regional de precipitação, em torno de $154 \mathrm{~mm}$, com uma diferença de $57,1 \mathrm{~mm}$ acima da média regional. Para o mês de fevereiro, a climatologia mensal da precipitação foi em torno de $182,5 \mathrm{~mm}$, e a média mensal regional de precipitação em torno de $154 \mathrm{~mm}$, com uma diferença de $28,5 \mathrm{~mm}$ acima da média. Através desses resultados, ficou evidente que um dos principais fatores responsáveis pela qualidade potencial da uva na região nordeste do Estado é, principalmente, a precipitação dos meses de janeiro e fevereiro.

O critério de definição da qualidade potencial da uva foi utilizado segundo a classificação das safras citadas por Mandelli et al. (2006). Dessa forma, os períodos de maturação classificados de alto potencial da uva no Estado foram os seguintes: 1981/ 1982, 1985/1986, 1990/1991, 1998/1999, 2001/2002, 2003/2004 e $2004 / 2005$. Os períodos de maturação classificados de baixo potencial da uva foram os seguintes: 1983/1984, 1987/1988, 1992/ 1993, 1994/1995, 1995/1996, 1997/1998 e 2002/2003.

Foram comparadas as climatologias mensais de precipitação em anos de alto e baixo potenciais da uva, para os meses de outubro a março, identificando o período de maior variação da precipitação na região nordeste do Estado. Percebese que a precipitação do período janeiro-fevereiro apresenta grande variação entre as safras de baixo e alto potenciais da uva, tornando-se necessário buscar as possíveis explicações para as variações pluviométricas desse período. Nesse sentido, passouse a buscar relações da precipitação desse bimestre com a TSM nos oceanos Atlântico e Pacífico.

Inicialmente, foram relacionadas as anomalias de TSM da região do Niño 3.4 no bimestre janeiro-fevereiro com as anomalias regionais de precipitação também desse período. Através do gráfico de dispersão (Figura 3), verifica-se a baixa correlação entre elas.

Fazendo-se uma análise da correlação das anomalias quantitativamente, percebe-se que, dos treze casos em que a anomalia da TSM foi positiva, em nove casos, a precipitação esteve acima da média, e os outros 4 casos, abaixo da média. Da mesma forma, ao se analisarem as anomalias negativas de TSM relacionadas com anomalias negativas de precipitação, a evidência da baixa relação entre elas é maior. Entre os onze casos de anomalia negativa de TSM, apenas em seis casos a anomalia de precipitação também foi negativa, e nos outros cinco casos, a anomalia de precipitação foi positiva, evidenciando-se a baixa relação entre essas duas variáveis.

Analisando-se as anomalias de TSM e as anomalias de precipitação qualitativamente, verificou-se que a correlação entre elas é da ordem de aproximadamente 0,16 , confirmando a baixa relação entre as chuvas de janeiro e fevereiro da região nordeste do Estado com a região do Niño 3.4. Percebe-se ainda, na Figura 3 , que as duas maiores estiagens ocorridas no bimestre janeiro e fevereiro, nessa região, aconteceram em anos de El Niños fracos (1991 e 2005), com anomalia negativa de precipitação de aproximadamente $148 \mathrm{~mm}$ e $180 \mathrm{~mm}$, respectivamente. Da mesma forma, maior excesso de precipitação ocorreu em um ano de La Niña fraca (1996), com anomalia da precipitação de aproximadamente $218 \mathrm{~mm}$. Por esses motivos, há a necessidade de se buscarem outras áreas do oceano que expliquem melhor essa relação com as chuvas nos meses de janeiro e fevereiro.

Ao verificar-se que a região do Niño 3.4 , sozinha, não explica as chuvas de janeiro e fevereiro da região nordeste do Estado, há a necessidade de serem identificadas outras áreas oceânicas, tanto no oceano Atlântico como no oceano Pacífico, que possam explicar melhor essa relação conjunta dos oceanos e a variação da precipitação naquela região.

A Figura 4 mostra as áreas dos oceanos Atlântico e Pacífico que apresentam coeficiente de correlação significativo com a precipitação nos meses de janeiro e fevereiro. A partir dessas áreas, foram extraídos 7 núcleos de correlação superior a $0,5 \mathrm{em}$ módulo (significativos a 1\%). Através da figura, identificam-se três áreas com correlação positiva e quatro áreas com correlação negativa. As áreas positivas estão relacionadas com a mesma direção do sinal das anomalias de TSM, e as anomalias de precipitação, nessa região, assim como as anomalias negativas de correlação, estão relacionadas na direção contrária entre si.

A Tabela 1 mostra as posições e os coeficientes de correlação dos 7 núcleos principais de maior correlação nos oceanos com a precipitação na região nordeste do Estado, representados pelas posições dentro da matriz de dados $\left(\mathrm{P}_{-} 342\right.$, P_267, P_179, P_116, P_301, P_174 e P_372). 
A Figura 5 mostra a relação entre a precipitação acumulada de janeiro e fevereiro e os escores do primeiro componente principal, gerado da série temporal de TSM dos sete núcleos de correlação. Os escores do primeiro componente principal foram suficientes para explicar grande parte da variação de TSM das sete áreas oceânicas escolhidas (variância explicada 87\%). Verificou-se que esses escores apresentam alta correlação com as chuvas de janeiro e fevereiro, com um coeficiente de correlação de 0,83 (Figura 5).

Além da alta correlação linear da precipitação com o primeiro componente, o gráfico mostra quantitativamente essa relação. Das quatorze vezes em que o escore do primeiro componente principal foi positivo, treze vezes a precipitação foi superior a $300 \mathrm{~mm}$, ou seja, superior à média acumulada de janeirofevereiro na região nordeste do Estado, indicando um percentual de relação de $93 \%$ com as chuvas de janeiro e fevereiro nessa região. Da mesma forma, das dez vezes em que os escores foram negativos, nove vezes a precipitação foi inferior à média, indicando um percentual de relação de $90 \%$ com as chuvas nesse mesmo período. Fica evidente que padrões positivos estão associados a anomalias positivas de precipitação. Da mesma forma, padrões negativos estão associados a anomalias negativas de precipitação na região nordeste do Estado.

Frente aos resultados, pode-se esperar que, a partir do sinal predominante do padrão principal, derivado da combinação de sete áreas oceânicas no período de janeiro-fevereiro, possibilite uma estimativa do sinal predominante da anomalia de precipitação regional e, conseqüentemente, uma tendência da qualidade potencial da uva na região nordeste do Rio Grande do Sul.
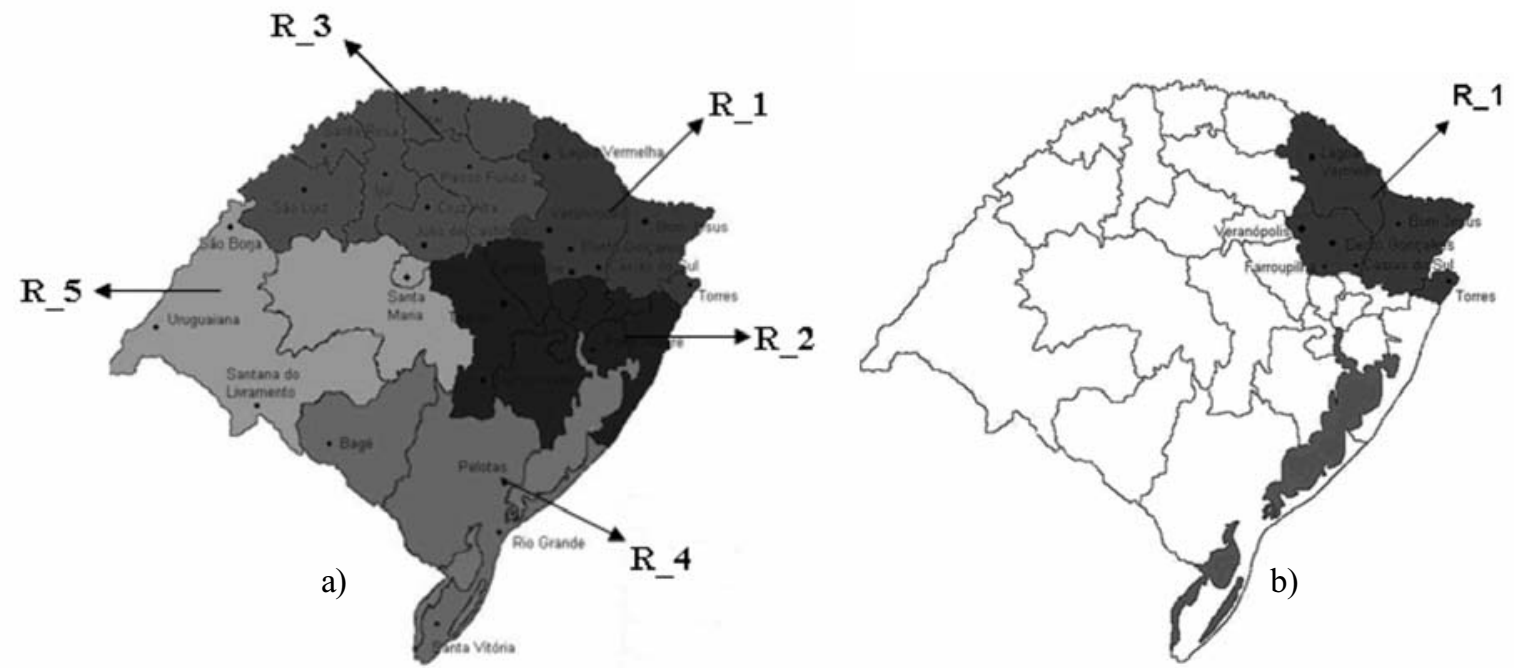

FIGURA 1- a) Regiões homogêneas de precipitação obtidas pelo método K-means, referente ao período de outubro a março de 1982 a 2005 para o Rio Grande do Sul; b) Região homogênea de precipitação escolhida, definida como nordeste.

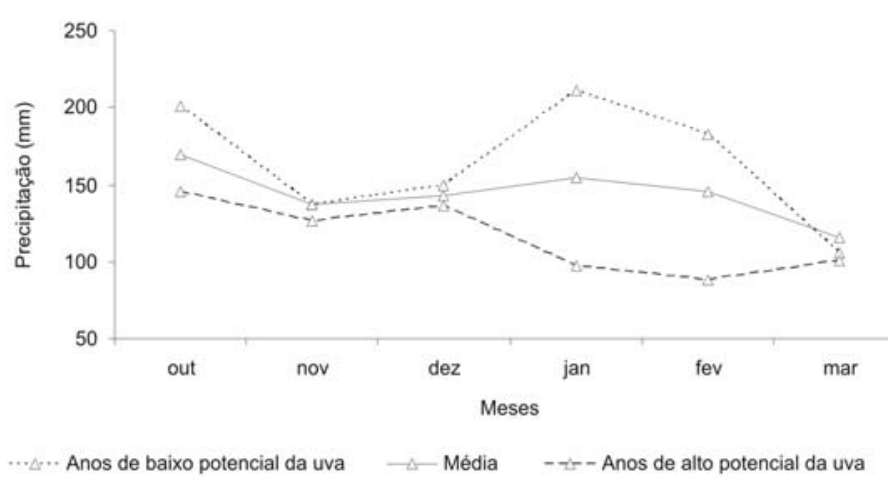

FIGURA 2 - Precipitação média mensal da região nordeste do Rio Grande do Sul, período de outubro a março de 1982 a 2005.

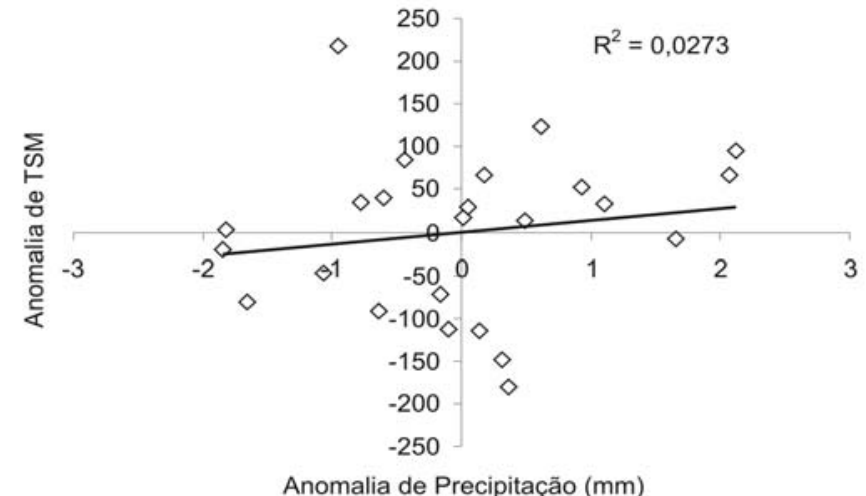

FIGURA 3 - Relação entre a anomalia da TSM média de janeiro e fevereiro na região do Niño 3.4 e a anomalia de precipitação total de janeiro e fevereiro na região nordeste do Estado, período de 1982 a 2005. 


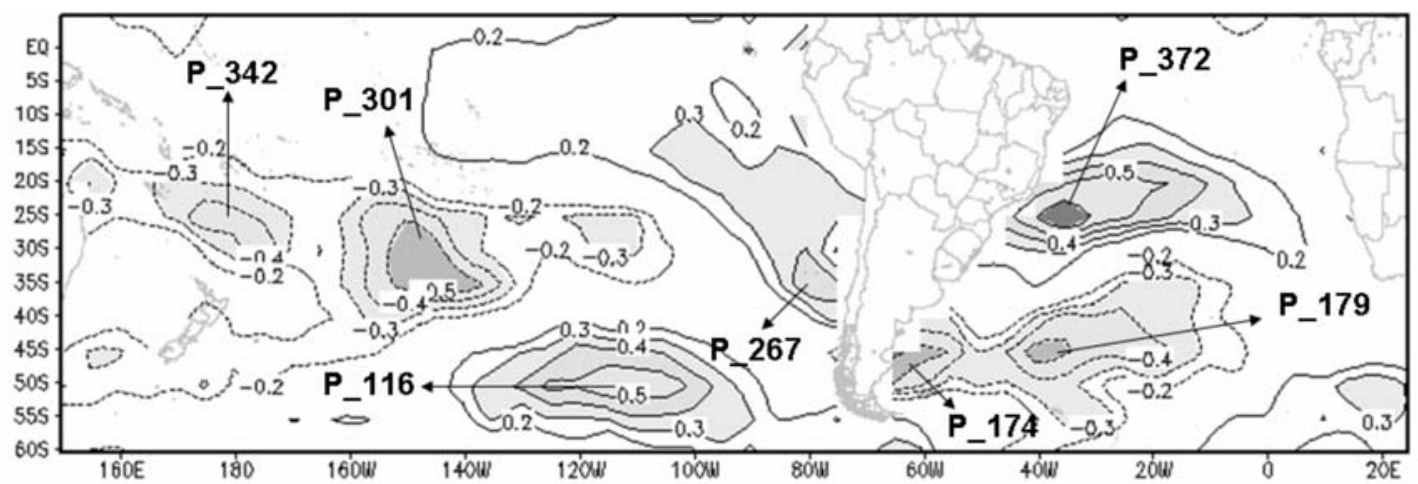

FIGURA 4 - Posições, nos oceanos Atlântico e Pacífico, dos 7 núcleos de maior correlação entre a TSM e a precipitação na região nordeste do Estado.

TABELA 1- Localização das posições de TSM escolhidas nos oceanos Atlântico e Pacífico, segundo as coordenadas latitude e longitude e seu coeficiente de correlação.

\begin{tabular}{cccc}
\hline Posições & Latitude & Longitude & $\begin{array}{c}\text { Coeficiente } \\
\text { Correlação }\end{array}$ \\
\hline 342 & $25^{\circ} \mathrm{S}$ & $180^{\circ} \mathrm{W}$ & 0,50 \\
267 & $30^{\circ} \mathrm{S}$ & $80^{\circ} \mathrm{W}$ & 0,50 \\
179 & $45^{\circ} \mathrm{S}$ & $40^{\circ} \mathrm{W}$ & 0,56 \\
116 & $50^{\circ} \mathrm{S}$ & $120^{\circ} \mathrm{W}$ & 0,59 \\
301 & $30^{\circ} \mathrm{S}$ & $145^{\circ} \mathrm{W}$ & 0,60 \\
174 & $45^{\circ} \mathrm{S}$ & $65^{\circ} \mathrm{W}$ & 0,63 \\
372 & $25^{\circ} \mathrm{S}$ & $40^{\circ} \mathrm{W}$ & 0,71 \\
\hline
\end{tabular}

\section{CONCLUSÕES}

1-A técnica de agrupamento K-means, aplicada sobre os escores dos componentes principais derivados da precipitação média regional de outubro a março, mostrou-se eficiente para separar 5 regiões homogêneas para o período de primavera-verão no Rio Grande do Sul.

2-Nos períodos de alto e baixo potenciais da uva na região nordeste, a precipitação mensal mostrou maiores diferenças nos meses de janeiro e fevereiro.

3-O primeiro padrão principal, derivado da combinação dos núcleos das 7 áreas oceânicas, mostrou que, ao tratar a variabilidade do oceano de forma conjunta nos oceanos Atlântico e Pacífico, aumenta o percentual de explicação da precipitação na região nordeste do Rio Grande do Sul, possibilitando também uma estimativa da qualidade potencial da uva nessa região.

\section{REFERÊNCIAS}

BERLATO, M. A. The Climate forecasting applications in the decision-making process for the Southern Region of Brazil. In: WORKSHOP REPORT ON ENSO AND SEASONAL TO INTERANNUAL CLIMATE VARIABILITY, SOCIO-ECONOMIC
IMPACTS, FORECASTING AND APPLICATIONS TO THE DECISION-MAKING PROCESS, 1992, Florianópolis: EPAGRI. v. 1, p. 14-16.

DIAZ, A. E.. et al. Relationship between precipitation anomalies in Uruguay and Southern Brazil and sea temperature in the Pacific and Atlantic oceans. Journal of Climate, Boston v.11, n.2, p. 251271, 1998.

DIAZ A. F.; STUDZINSKI, C. D. Rainfall anomalies in the Uruguay-Southern Brazil region related to SST in Pacific and Atlantic oceans using canonical correlation analysis. In: CONGRESSO BRASILEIRO DE METEOROLOGIA, 8., CONGRESSO LATINO - AMERICANO DE METEOROLOGIA, 2., Belo Horizonte, 1994. Anais.. São José dos Campos: INPE. v.1, p. 498-501.

MANDELLI, F. et al. Classificação Climática dos períodos de maturação das safras vitícolas de 1977 a 2003. Boletim Técnico Embrapa Uva e Vinho, 2006. Disponível em: < http:// www.cnpuv.embrapa.br/>. Acesso em: 21 jan. 2007.

MARQUES, J. R. Variabilidade espacial e temporal da precipitação pluvial no Rio Grande do Sul e sua relação com indicadores oceânicos. 2005. 209f. Tese (Doutorado em Fitotecnia) - Universidade Federal do Rio Grande do Sul, Porto Alegre, 2005.

WESTPHALEN, S. L. Bases ecológicas para determinação de regiões de maior aptidão vitícola no Rio Grande do Sul. In: SIMPÓSIO LATINO-AMERICANO DE LA UVA Y DEL VINO, 1977, Montevideo: Ministério de Industria y Energia, Laboratório Tecnológico del Uruguay, 1977, v.1, p.89-101. (Cuaderno Tecnico, 38). 